\title{
The analysis of Upper tract Urinary Stone Composition in HIV-positive Patients: a single center experience
}

\section{Wu Menghua}

Beijing You'an Hospital, Capital Medical University

\section{Zheng Xin}

Beijing You'an Hospital, Capital Medical University

\section{Fan Bohan}

Beijing Chaoyang Hospital, Capital Medical University

\section{Zhang Yu}

Beijiing You'an Hospital, Capital Medical University

\section{Zhao Jimao ( $\nabla$ zhaojimao@foxmail.com )}

Beijing Friendship Hospital, Capital Medical University https://orcid.org/0000-0002-5158-4363

\section{Research article}

Keywords: urinary calculi, stone composition, HIV-infected, Negative邓UA囚HARRT

Posted Date: May 22nd, 2020

DOl: https://doi.org/10.21203/rs.3.rs-29836/v1

License: (c) (1) This work is licensed under a Creative Commons Attribution 4.0 International License. Read Full License 


\section{Abstract}

Background: To appraise the most common stone composition, serum and urinary biochemical features in HIV-positive patients with urolithiasis.

Methods: We retrospectively searched the records of HIV-positive patients in our stone registry. A retrospective study was generated from our registry. Collecting statistics about fundamental indexes and analyzing them is our basic procedure. Medications were considered. Groups were compared using the Student $t$ and chi-square/Fisher exact tests with significance considered at $p<0.05$.

Results: In total of 101 patients were included age, gender, Body Mass Index(BMI), serum calcium, serum uric acid(UA), stone position, and operative method were statistically described. The serum UA of HIVpositive group was higher than that of the negative group $(P=0.028)$. Patients with HIV-infected had a higher incidence of calcium oxalate dehydrate $(\mathrm{CaODH})(\mathrm{p}<0.001)$. The evaluation of overall stone composition showed that the number of CaODH was higher in HIV-positive group $(21.4 \%$ vs $8.5 \%$, $p<$ $0.001)$. There was no significant difference in single calcium oxalate monohydrate $(\mathrm{CaOMH})(p=0.146)$, and UA $(p=0.7)$ between the two groups.

Conclusions: In our study the highest stone proportion in HIV patients is calcium oxalate (CaO). HIV positive patients should pay attention to the diet, especially the food containing more calcium. In HIVpositive patients, the level of serum UA should be controlled accordingly. In the development of highly active antiretroviral therapy (HARRT), for low-risk patients, it may not be necessary to consider too much the factors of protease inhibitors (PIs) and other drugs forming urinary calculus.

\section{Background}

Urinary stone is the most common disease of the urology. China is one of the most widespread countries with a high morbidity rate of urolithiasis patients. In China, the disease approximately affects $4 \%$ of population and increases steadily during the recent 20 years, due to high calories intake from dietary and lack of exercise in lifestyle ${ }^{[1]}$. With its high morbidity rate, urologists indeed attach importance to the disease. Sometimes urinary stones can lead to nephrosis, renal colic and reduce the quality of life. It may be harmful to patients' renal function ${ }^{[2]}$. As the complexity of the pathogenesis, the accomplishment of early clinical prevention and early diagnosis is difficult. Recent studies reported the stone composition in Chinese patients from different regions mainly include $\mathrm{CaO}$ and $\mathrm{UA}^{[3,4]}$. As a surgical diagnosis and treatment center of the epidemic disease hospital which is the biggest one in China, we found the number of stone patients with HIV-positive had increased gradually in recent years. Analyzing the composition of urinary stones in a region is of great significance for the diagnosis, treatment, and prevention of calculi. Currently, infrared spectroscopy is the standard method used to analyze urinary stone components. Some studies reported that a few urinary stones were composed of protease inhibitors (PIs ${ }^{[5,6]}$. But these studies contained a few cases and cannot explain the widespread stone compositions in HIV-positive patients. As our knowledge, no investigation has been performed about the stone composition in HIV 
infected patients. We determined to compare the stone composition between HIV infected and negative patients, and to explore the underlying factors.

\section{Material And Methods Study Design}

After the approval of the institutional review committee,All patients in this study provided written informed consent before treatment. We retrospectively analyzed and collected the data of patients from January 2013 to January 2019. This study only included cases of urinary calculi that had stone composition analysis. Stone samples were obtained by surgery. The cases included in this study are divided into two groups, the one includes HIV-infected patients, and another includes HIV-negetive patients. At last we analyzed the stone composition. The stone composition categories includes $\mathrm{CaOMH}$, $\mathrm{CaODH}$, calcium phosphate (CaPh), UA, struvite, and cystine.

\section{Metabolic And Laboratory Evaluation}

In this study, 32 HIV-positive patients and 69 negetive patients were included. General information includes gender, age, BMI, etc. HIV patients recorded the regular HARRT regimen. Serum indexes, including serum calcium and UA were collected. The HIV-positive patients have been in regular HARRT. To compare the data between serum indexes and stone composition. We analyzed the related datas in the two groups.

\section{Stone Composition}

The stone components were divided into the following categories: $\mathrm{CaOMH}, \mathrm{CaODH}$. $\mathrm{CaPh}, \mathrm{UA}$, struvite, cysteine. The stones were washed with water and placed in an oven at $70 \mathrm{C}-100 \mathrm{C}$ to dry. The stone powder $(1 \mathrm{mg})$ was mixed with dry potassium bromide $(200 \mathrm{mg})$, and then the mixture was ground with an agate mortar. Liir-20 type of stone was used to analyze the composition of stone. Stone composition was analyzed by Liir-20 transform infrared spectroscopy and expressed as the amount of each component in the stone. Finally, the computer draws the spectrum map and analyzes the stone composition automatically. Through manual analysis of the spectrogram, the results are verified. If necessary, polarizing microscope and chemical analysis are also attached. We compared the proportion and composition of each stone component between the experimental group and the control group. We pooled the proportion of stone components in each patient. In this study, it was determined that more than $70 \%$ of the stone component was pure stone. In addition, the incidence of pure stone and mixed stone were compared between the two groups.

\section{Statistical Analysis}


Statistical analysis was performed using SPSS ${ }^{\circledR}$, version 22. Results are shown as the mean \pm SD. Groups were compared using the Student t-test for numerical variables and the chi-square or Fisher exact test for categorical variables. $\mathrm{P}<0.05$ was considered statistically significant.

\section{Results}

From January 2013 to January 2019, we screened 215 patients with upper urinary tract calculi in our center and excluded 114 patients without a calculus component analysis. A total of 17 cases underwent percutaneous nephrolithotripsy and 84 cases underwent ureteroscopy. According to whether they were combined with HIV, they were divided into two groups, including $32 \mathrm{HIV}$ positive patients and 69 negative patients. 32 HIV positive patients who had HARRT. The HARRT mainly contain zidovudine (AZT), lamivudine (3TC), lopinavir/ritonavir(LPV/r), navirapin(NVP), efivirenz(EFV), raltegravir(RAL), tenofovir(TDF). The ART of HIV group contains 9 patients with AZT + 3TC + LPV / r (29\%); 6 patients with $A Z T+3 T C+N V P(19.4 \%) ; 2$ patients with AZT + 3TC + EFV (6.4\%); 2 with AZT + 3TC + RAL (6.4\%); 3 with $A Z T+T D F+L P V / r(6.4 \%)$ and 10 with TDF + 3TC + LPV / r (32.2\%). The age 849.06 VS $54.46 p=0.528 \rrbracket$ gender, BMI (27.53 VS $26.77 p=0.388)$, serum calcium ( 2.29 VS $2.30 p=0.615)$, potassium (4.02 VS 4.16 $p=0.035)$, sodium (140.09 VS $139.67 p=0.386)$, creatine (76.4 VS $77.6 p=0.752)$, ALB (43.6 VS 40.5), Urine Ph (5.98 VS $5.91 p=0.482)$, serum UA (349.06 VS $306 p=0.028)$, stone position, and operation mode were statistically described. The serum UA of HIV positive group was higher than that of HIV negative group $(p=0.028)$. No significant statistical significance was found in other general data (Table 1). 
Table 1

Demographic data on 101 patients with and without HIV-infected

\begin{tabular}{|c|c|c|c|}
\hline & HIV & Neg & $\begin{array}{l}P \\
\text { Value }\end{array}$ \\
\hline No. pts & 32 & 69 & \\
\hline No. men/women & $29(90.6) / 3(10.4)$ & $34(49.2) / 35(51.8)$ & \\
\hline Mean \pm SD age(range) & $49.06 \pm 13.75(33-73)$ & $54.46 \pm 12.45(28-81)$ & 0.528 \\
\hline Mean \pm SD kg/هBMI(range) & $\begin{array}{l}27.53 \pm 4.00(15.17- \\
34.89)\end{array}$ & $\begin{array}{l}26.77 \pm 4.20(20.70- \\
36.16)\end{array}$ & 0.388 \\
\hline \multicolumn{4}{|l|}{$\begin{array}{l}\text { Mean } \pm \text { SD laboratory serum } \\
\text { evaluation(range) }\end{array}$} \\
\hline Calcium(mg/dl) & $2.29 \pm 0.12(2.05-2.57)$ & $2.30 \pm 0.13(2.00-2.70)$ & 0.615 \\
\hline Urinary acid(umol/L) & $\begin{array}{l}349.06 \pm 95.95(177- \\
608)\end{array}$ & $\begin{array}{l}306.70 \pm 85.29(142- \\
506)\end{array}$ & 0.028 \\
\hline Potassium(mmol/L) & $4.02 \pm 0.28(3.53-4.86)$ & $4.16 \pm 0.78(3.32-5.4)$ & 0.035 \\
\hline Sodium(mmol/L) & $\begin{array}{l}140.09 \pm 2.25(135.6- \\
1454)\end{array}$ & $139.67 \pm 2.30(135.2-$ & 0.386 \\
\hline (a) & $76.4+142(51-110)$ & $744.5)$ & 0.152 \\
\hline $\operatorname{ALB}(g / L)$ & $76.4 \pm 14.3(54-110)$ & $77.6 \pm 22.0(49-190)$ & $\begin{array}{l}< \\
0.001\end{array}$ \\
\hline Stone CT value (range) & $43.6 \pm 4.9(35-52.7)$ & $40.5 \pm 3.4(34.3-46.4)$ & 0.091 \\
\hline Urine $\mathrm{Ph}$ & $\begin{array}{l}611.84 \pm 259.68(261- \\
1187)\end{array}$ & $\begin{array}{l}721.49 \pm 361.58(194- \\
1423)\end{array}$ & 0.482 \\
\hline $\mathrm{CD}^{+}$ & $5.98 \pm 0.35(5.5-6.5)$ & $5.91 \pm 0.91(5-7.5)$ & 0.855 \\
\hline Stone position(\%) & $520.4 \pm 241.6(49-926)$ & $14(20.3)$ & 0.524 \\
\hline Renal & $7(21.8)$ & $55(79.7)$ & 0.726 \\
\hline Ureter & $25(78.2)$ & 11 & 0.465 \\
\hline No. treatment modality (\%) & 6 & 58 & \\
\hline Percutaneous nephrolithotomy & 26 & & \\
\hline
\end{tabular}

Demographic characteristics (age, gender, BMI) were no significance in the 101 patients (Table 1). The two groups have no significant difference in age $(p=0.528)$, sex $(p=0.388)$, and BMI $(p=0.615)$. The serum UA of HIV positive group was higher than that of HIV negative group $(P=0.028)$. No significant statistical significance was found in other general data.

\section{Stone Composition}


We identified 32 patients with HIV-infected and 69 negative. There was no significant difference in $\mathrm{CaOMH}$ composition between the two groups $(38.4 \%$ vs $48.9 \%, \mathrm{P}=0.119)$. Patients with HIV-infected had a higher incidence of $\mathrm{CaODH}(\mathrm{p}<0.001)$. The evaluation of overall stone composition showed that the number of $\mathrm{CaODH}$ was more in HIV group $(21.4 \%$ vs $8.5 \%, \mathrm{p}<0.001)$ (Table 2). Although the proportion of $\mathrm{CaODH}$ in the total stone is higher than that of the negative group, Pure $\mathrm{CaODH}$ stone is not found in HIV, but as a secondary component, there is a higher proportion (Fig. 1). Moreover, there is no difference in $\mathrm{CaOMH}$, these results suggest that dietary or other factors related to calcium calculi should be taken into account in HIV patients.. No obvious abnormality was found in Struvite and Cystine. There was no significant difference in single $\mathrm{CaOMH}(56 \%$ vs. $52, \mathrm{p}=0.146)$, $\mathrm{CaPh}(5 \%$ vs. $2 \% \mathrm{p}=0.069)$ and UA ( $39 \%$ vs. $40 \%, p=0.7)$ between the two groups. There were 62 cases of single stone $(>70 \%)$, including 18 cases in HIV group and 44 cases in negative group. There was no significant difference between the two groups $(p=0.727)$ (Table 3). At last, we analyzed the main components of stones in different HAART treatments (Table 4) .

Table 2

Overall stone composition data in patients with HIV-infected VS patients with Negtive

\begin{tabular}{|llll|}
\hline & HIV & Neg & p Value \\
\hline No. pts & 32 & 69 & \\
$\begin{array}{l}\text { Mean } \pm \text { SD \% stone } \\
\text { composition(range) }\end{array}$ & & & \\
CaOMH & $38.4 \pm 26.9(0-$ & $48.9 \pm 33.3(0-100)$ & 0.119 \\
\hline CaODH & $100)$ & & \\
\hline CaPh & $21.4 \pm 22.4(0-75)$ & $8.5 \pm 11.9(0-50)$ & $<0.001$ \\
\hline UA & $5.2 \pm 13.9(0-73)$ & $4.6 \pm 11.4(0-35)$ & 0.843 \\
\hline Struvite & $24.8 \pm 35.7(0-93)$ & $33.5 \pm 31.7(0-100)$ & 0.217 \\
\hline Cystine & $1.5 \pm 5.5(0-100)$ & $3.9 \pm 14.4(0-100)$ & 0.364 \\
\hline
\end{tabular}


Table 3

Pure Stone composition data in patients with HIV-infected and negative.

\begin{tabular}{|llll|}
\hline & HIV & Neg & p Value \\
\hline No.mixed(\%) & $14(43.8)$ & $25(36.2)$ & \\
\hline No. 70\% or greater pure (\%) & 18 & 44 & 0.727 \\
\hline CaOMH & $10(56)$ & $23(52)$ & 0.146 \\
\hline CaODH & $0(0)$ & $1(2)$ & 0.069 \\
\hline CaPh & $1(5)$ & $1(2)$ & 0.051 \\
\hline UA & $7(39)$ & $15(40)$ & 0.70 \\
\hline Struvite & $0(0)$ & $1(2)$ & 0.49 \\
\hline Cystine & $0(0)$ & $1(2)$ & 0.49 \\
\hline
\end{tabular}

Table 4

Main stone composition in different HAART group

\begin{tabular}{|lllllll|}
\hline & $\begin{array}{l}\text { AZT+3TC } \\
+\mathrm{LPV} / \mathbf{r}\end{array}$ & $\begin{array}{l}\text { AZT + } \\
\text { 3TC+NVP }\end{array}$ & $\begin{array}{l}\text { AZT + } \\
\text { 3TC+EFV }\end{array}$ & $\begin{array}{l}\text { AZT + } \\
\text { 3TC+RAL }\end{array}$ & $\begin{array}{l}\text { AZT+ TDF } \\
+ \text { LPV } / \mathbf{r}\end{array}$ & $\begin{array}{l}\text { TDF + 3TC+ } \\
\text { LPV / } \mathbf{r}\end{array}$ \\
\hline $\begin{array}{l}\text { Main } \\
\text { composition }\end{array}$ & & & & & & \\
\hline $\mathrm{CaOMH}$ & 3 & 2 & 1 & 2 & 1 & 5 \\
\hline $\mathrm{CaODH}$ & 1 & 0 & 1 & 0 & 0 & 1 \\
\hline $\mathrm{CaPh}$ & 1 & 0 & 0 & 0 & 0 & 0 \\
\hline $\mathrm{UA}$ & 4 & 4 & 0 & 0 & 2 & 4 \\
\hline & 9 & 6 & 2 & 2 & 2 & 10 \\
\hline
\end{tabular}

\section{Discussion}

As the one of the largest surgery center for HIV-positive patients in China, most of HIV positive urinary calculi patients in north China have came to our center for the cure. We found that the number of HIVpositive patients with stones had been increasing gradually in recent years. So we conducted the study to explore the reason and an possible way for prevention.

There are great differences in stone composition of different regions in the world. Amir's study of the stone composition in the Arab region showed that $\mathrm{CaOMH}$ is the most common one, accounting for almost $85 \%$ of all the patient ${ }^{[7]}$. Tyson ${ }^{[8]}$ analyzed stones in Northern Ireland and also found that there was a high proportion of stones containing $\mathrm{CaO}$ (94.4\%). Girişgen ${ }^{[9]}$ analyzed the stones of children in the 
central and western regions of Turkey, and found that $\mathrm{CaOMH}$ was the most common components, which might be related to diet habits. The prevalence of kidney stones in the United States in 2007-2010 is markedly higher than when last measured in 1988-1994.

In recent years, with the change in people's life and diet, the incidence rate of urinary calculi in China is also increasing ${ }^{[3,10]}$. Because of the difference in geography, the composition of stones are also different. As known, the main composition of stones found in China is $\mathrm{CaO}$. Ma systematically evaluated the multicenter studies in the north, East, central, South and southwest of China ${ }^{[4]}$, and found that there were some differences in the material and element composition of urinary calculi in different regions. Zhang analyzed the composition of 3684 patients in the east of Shandong Province and found that $\mathrm{CaO}$ stone appeared most frequently. In Southwest China, the propotion of $\mathrm{CaO}$ stone is the highest, while in South China that of UA stone is the highest ${ }^{[3]}$. In our study, we found that the CAO is the most one in the cohort, which is similliar to the propotions reported in HIV-negative patients before. As the cohort contains patients from both north and south of China, we still found certain propotion of UA which was also similliar to studies in HIV-negative patients.

UA is a common composition, accumulation about $40 \%$ in US, and $30 \%-40 \%$ in china. ${ }^{[3,10]}$ UA stone are already reported in many regions in large sample of Chinese data. In addition, the clinical features were highly correlated with stone type and anatomical location, but not with distribution area. It conforms to the current situation of stone in China.

Some studies suggested that higher serum UA level does not necessarily lead to UA stones. Patients with gout suppose to have higher incidence rate in UA urinary calculi. But Marchini found UA stones are the most common pure stone composition in patients with gout but $48 \%$ have nonuric acid stones. The serum UA level is just a cause or a risk factor for the formation of UA stones. Most have other metabolic riskfactors for stone formation. Yu and Gutman have reported that urinary stones were ascribed to hyperuricemia. The number of cases included in this study is relatively small, which may affect the results. Song also reported the relationship between serum UA level and metabolic abnormalities, among which 159 twins' differences in UA level were also related to metabolic abnormalities ${ }^{[11]}$. And for HIV positive patients, there is study repoting the possible influence of HAART to induce the accumulation of UA. Pirro ${ }^{[12]}$ reported a study of 250 HIV-positive patients that after HAART treatment for at least 6 months, the serum UA level of the patients increased. The analysis indicated that it might be related to the insufficiency of endothelial cell function after HARRT. The study also found that the increase of serum UA level often occurred after receiving HAART, but it still needs a large number of prospective studies to verify. It can also be understood that the high level of UA in HIV positive group and urinary calculi may be related to the metabolic abnormality caused by antiviral treatment.

In our study, we found that the serum UA level of the HIV positive group is higher than that of HIV negative group, but there is no significant difference in the propotion of UA stones between two groups. 
Because of the specific HARRT regime, evaluation of the stone composition of HIV-positive patient is essential.Acoording to previous studies, kidney stones were found to be more common in HIV positive patients taking protease inhibitors (PIs) ${ }^{[13]}$,and the main composition is the component of the medicine. This was mainly in the cases with indinavir, and this was also found in the cases with atazanavir. Other Pls such as nelfinavir, amprenavir, saquinavir, ritonavir and darunavir have also been reported to cause urolithiasis or to crystallize in urine. Ritonavir urolithiasis have been reported in several cases ${ }^{[14]}$. Little is known regarding the pathophysiology of renal colic in patients taking Pls. Previous studies have suggested that impaired hepatic function could result in increased renal elimination, as hepatic metabolism is important for many of these Pls. Rockwood ${ }^{[13]}$ found Individuals with drug genetic predisposition to slower ritonavir-boosted atazanavir (ATZ/r) metabolism may increase the risk of stone formation due to higher ATZ/r-related levels. Previous studies have shown that impaired liver function may lead to increased renal clearance, because liver metabolism is important for many of these PIs. However, despite a large number of reports on HIV PIs related kidney stones, the formation mechanism of these kidney stones is not completely clear. In a retrospective study, only $28 \%$ of indinavir treated patients with kidney stones had stones containing indinavir ${ }^{[15]}$. The other patients who did not take indiavir had stones containing calcium oxalate, ammonium urate and UA, and some had various metabolic abnormalities including low-carbon, high oxaluria and high calcium urine. Vassallo reported a case in which the debris found after lithotripsy was mainly Raltegravir ${ }^{[16]}$. The plasma and urine concentrations of Raltegravir are at normal limits, making it unlikely that the dose will be inadequate. Only patients with a history of urolithiasis should be cautious with the prescription. But In our study, we analized 32 cases and we did not discover HAART medicine related stones composition ${ }^{[5]}$.

And in the study we included that there were 21 people taking LPV / $r$, but no drug stone component was found. We suggested the that the reason might be related the HIV-positive group's the basic normal liver and kidney functions. The metabolization in HIV-positive group may not be affected by the HAART. The occurrence of stone also may occur before HIV infection, because after HIV infection, the relevant examination was relatively in advance, so kidney stone was found earlier. It may indicate that we can make antiviral program for low-risk patients without considering the factors of PI drugs leading to stones.

The comparison of pure stones in our study, calcium oxalate dihydrate in HIV positive group was higher than that in negative group. There may be a higher risk of complications from taking antiretroviral drugs with urinary stones.

In addition, we also analized the CD4 count of HIV positive group. Although there were 4 patients less than 200 , no serious urinary tract infection was found after the operation, which may be related to the preoperative full evaluation, control of operation time and the prevention and application of antibiotics. In terms of operation mode, the HIV group and the normal group were mainly operated by ureteroscopy with less trauma, and PCNL was used relatively small, but it may also be related to the relatively small number of cases. The next step may be to compare the operation outcome. 
However, this case-control trial increases the possibility of prognostic differences between HIV and negative groups. There was no significant difference in age, gender and BMI. The advantages of our study include the comparison of stone composition. However there is still a need for further study and design of randomized controlled trials. We should have more patients to go in for studies. The study will be continued in the future.

We have determined to pay more attention to the HIV-positive patients with urinary calculi. Above all, we need to collaborate more details to analyze. At last, this study retrospective analysis of single center cases in our hospital, case-control study of stone composition analysis for patients with HIV infection and normal patients, hoping to observe some situations, and provide some data support for clinical early prevention.

\section{Conclusion}

In our study the highest stone proportion in HIV patients is calcium calculi. HIV positive patients should pay attention to the diet, especially the food containing more calcium. In HIV patients, the level of serum uric acid should be controlled accordingly. Although the formation of uric acid calculus is not necessarily in direct proportion to the level of uric acid, in the development of HARRT, for low-risk patients, it may not be necessary to consider too much the factors of Pls and other drugs forming urinary calculus.

\section{Declarations}

\section{Ethics approval and consent to participate}

The experimental protocol was established, according to the ethical guidelines of the Helsinki Declaration and was approved by the Human Ethics Committee of Beijing You'an Hospital. Written informed consent was obtained from individual or guardian participants.

\section{Consent of publication}

Not applicable.

\section{Availability of data and material}

All data generated or analysed during this study are included in this published. Article

\section{Competing Interests}

The authors declare that they have no competing interests.

\section{Funding}

Not applicable. 
Authors' contributions

Wu Menghua and Fan Bohan collected patients data. Wu Menghua and Zheng Xin analyzed and interpreted the patient data. Wu Menghua and Zheng Xin were major contributors in writing the manuscript. All authors read and approved the final manuscript.

\section{Acknowledgements}

We appreciated Song Jian for revising grammer errors.

\section{References}

1. Zeng Q. and He Y, Age-specific prevalence of kidney stones in Chinese urban inhabitants. Urolithiasis, 2013. 41(1): p. 91-3.

2. Skolarikos A, Straub M, Knoll T, Sarica K, Seitz C, Petřík A, Türk C, Metabolic evaluation and recurrence prevention for urinary stone patients: EAU guidelines. Eur Urol, 2015 67: p. 750-763.

3. Zhang X, Ma J, Wang N, Lin C. Urinary stone composition analysis of $\mathbf{3 6 8 4}$ patients in the eastern Shandong region of China. J Int Med Res, 2019. 28.

4. Ma RH, Luo XB,Li Q,Zhong HQ. Systemic analysis of urinary stones from the Northern, Eastern, Central, Southern and Southwest China by a multi-center study. BMC Urology, 2018. 18(1).

5. Izzedine H. Lescure FX. Bonnet F. HIV medication-based urolithiasis. Clinical Kidney Journal, 2014. 7(2): p. 121-126.

6. Wirth GJ, Teuscher J, Graf JD, Iselin CE. Efavirenz-induced urolithiasis. Urol Res, 2006. 34: p. 288289.

7. Amir A. Matlaga BR.Ziemba JB.Sheikh S. Kidney stone composition in the Kingdom of Saudi Arabia. Clin Nephrol, 2018. 89(5): p. 345-348.

8. Matthew T, Nathen G, Laura M. Renal and Ureteric Stone Composition A five year retrospective study for Northern Ireland. Ulster Med J, 2019. 88: p. 21-24.

9. Girişgen I YS., Karcılı K, Becerir T, Evaluation of the composition of urinary tract stones in children from the Inner Western Anatolian Region in Turkey. Turk J Urol, 2020. 46: p. 152-158.

10. He Z, Jing Z, Jing-Cun Z, Chuan-Yi H , Fei G. Compositional analysis of various layers of upper urinary tract stones by infrared spectroscopy. Exp Ther Med, 2017. 14: p. 3165-3169.

11. Song YM and K Lee, Genetic and Environmental Influences on the Associations Between Uric Acid Levels and Metabolic Syndrome Over Time. Metabolic Syndrome and Related Disorders, 2018. 16(6): p. 299-304.

12. Pirro M, Bianconi V, Schiaroli E, Francisci D, Mannarino MR, Bagaglia F.et al. Elevated serum uric acid levels are associated with endothelial dysfunction in HIV patients receiving highly-active antiretroviral therapy. Atherosclerosis, 2018. 272: p. 101-107. 
13. Rockwood N 1, Mandalia S, Bower M, Gazzard B, Nelson M. Ritonavir-boosted atazanavir exposure is associated with an increased rate of renal stones compared with efavirenz, ritonavir-boosted lopinavir and ritonavirboosted darunavir. AIDS, 2011(25): p. 1671-1673.

14. Doco-Lecompte T, Garrec A, Thomas L, Trechot P, May T, Rabaud C. Lopinavir-ritonavir (Kaletra) and lithiasis: seven cases. AIDS, 2004. 16: p. 705-6.

15. Nadler RB, Rubenstein JN, Eggener SE, Loor MM, Smith ND.The etiology of urolithiasis in HIV infected patients. Am Urolog Assoc, 2003. 169: p. 475-477.

16. Vassallo M, Dunais B, Naqvi A, Garaffo R, Durant J. Raltegravir-induced nephrolithiasis: a case report. AIDS, 2012. 26: p. 1323-1324.

\section{Figures}
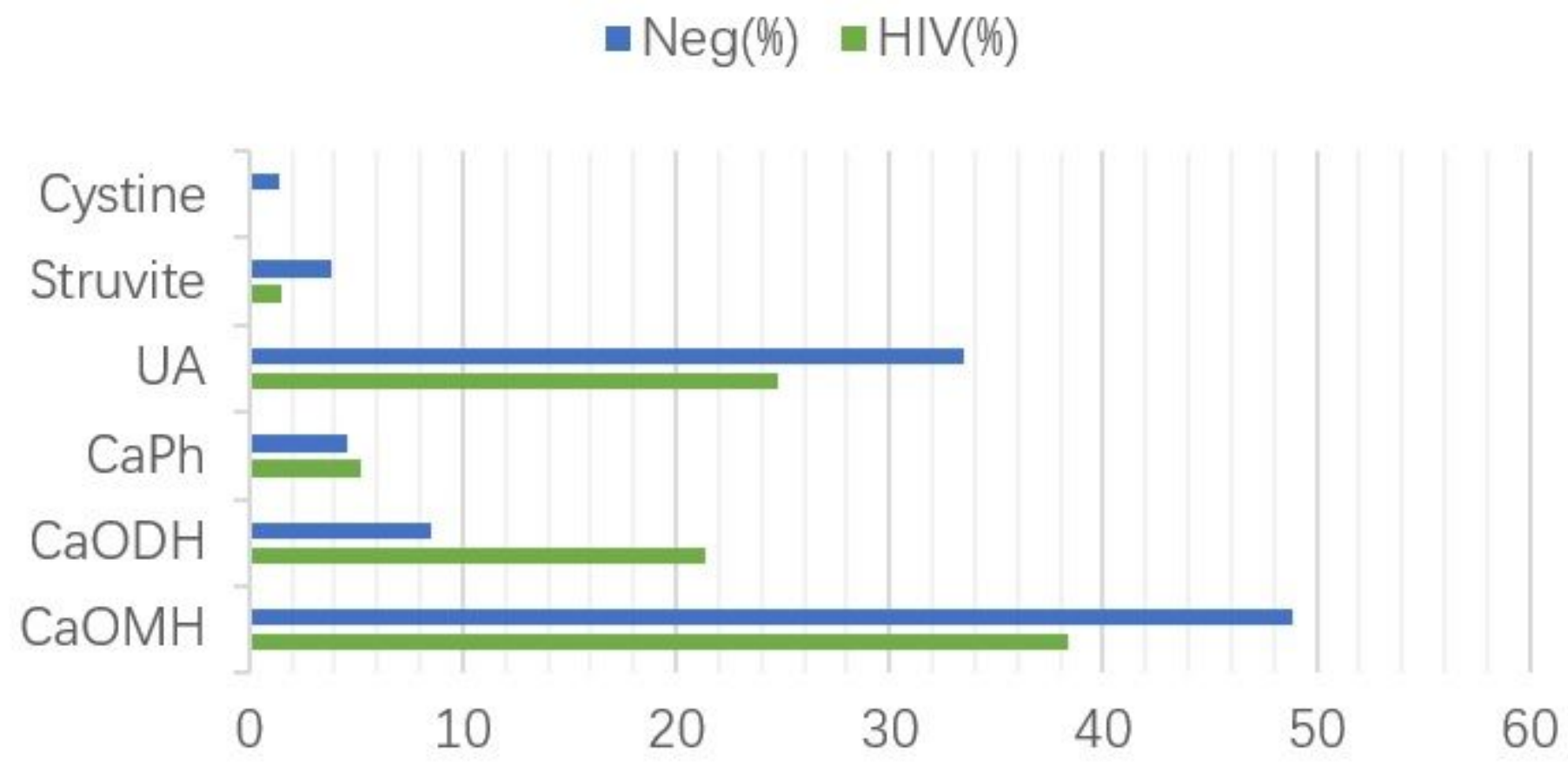

Figure 1

Overall stone component rate 